OPEN ACCESS

Edited by:

Alok Raghav,

Ganesh Shankar Vidyarthi Memorial

Medical College, India

Reviewed by:

Jamal Ahmad,

Aligarh Muslim University, India

Mohammad Zubair,

University of Tabuk, Saudi Arabia

${ }^{*}$ Correspondence:

Jaffer Shah

jaffer.shah@kateb.edu.af

tThese authors share first authorship

Specialty section:

This article was submitted to

Clinical Diabetes,

a section of the journal

Frontiers in Public Health

Received: 24 December 2021

Accepted: 27 January 2022

Published: 25 February 2022

Citation:

Sayed Ahmed HA, Mohamed SF, Elotla SF, Mostafa M, Shah J and

Fouad AM (2022) Psychometric

Properties of the Arabic Version of the

Problem Areas in Diabetes Scale in

Primary Care.

Front. Public Health 10:843164

doi: 10.3389/fpubh.2022.843164

\section{Psychometric Properties of the Arabic Version of the Problem Areas in Diabetes Scale in Primary Care}

\author{
Hazem A. Sayed Ahmed ${ }^{1 \dagger}$, Samar Farag Mohamed ${ }^{1 \dagger}$, Sally Fawzy Elotla ${ }^{2}$, Mona Mostafa ${ }^{3}$, \\ Jaffer Shah ${ }^{4 *}$ and Ahmed Mahmoud Fouad ${ }^{2}$ \\ 'Department of Family Medicine, Faculty of Medicine, Suez Canal University, Ismailia, Egypt, ${ }^{2}$ Department of Public Health, \\ Occupational and Environmental Medicine, Faculty of Medicine, Suez Canal University, Ismailia, Egypt, ${ }^{3}$ Department of \\ Internal Medicine, Faculty of Medicine, Suez Canal University, Ismailia, Egypt, ${ }^{4}$ Medical Research Center, Kateb University, \\ Kabul, Afghanistan
}

Background: The Problem Areas in Diabetes (PAID) scale is a reliable and valid tool that is widely used for diabetes-distress screening, but the Arabic version of the scale lacks validity and reliability analysis in primary healthcare $(\mathrm{PHC})$ patients. Our study aimed to evaluate the psychometric properties of the Arabic version of the PAID (AR-PAID) scale among Egyptian patients with type 2 diabetes mellitus (T2DM) in PHC settings.

Methods: We conducted a cross-sectional study on a convenience sample of 200 patients from six rural PHC settings in the Ismailia governorate. The confirmatory factor analysis (CFA) was performed to test the goodness-of-fit to the predefined models of the PAID. Convergent construct was evaluated through correlations with the Arabic versions of the Patient Health Questionnaire 9 (PHQ-9), Generalized Anxiety Disorder Scale (GAD7), and 5-item World Health Organization Well-Being Index (WHO-5), additionally glycated hemoglobin ( $\mathrm{HbA} 1 \mathrm{c})$ levels. Discriminant validity was evaluated through associations with patients' sociodemographic and clinical characteristics. Reliability was evaluated through internal consistency (Cronbach's $\alpha$ ) and test-retest reliability analysis (intraclass correlation coefficient, ICC).

Results: The CFA demonstrated the best fit for a four-factor model. The AR-PAID was significantly correlated with the following measures: PHQ-9 (rho $=0.71, p<0.001$ ), GAD-7 (rho $=0.50, p<0.001$ ), WHO-5 (rho $=-0.69, p<0.001$ ), and HbA1c (rho $=0.36, p<0.001$ ), supporting sound convergent validity. Discriminant validity was satisfactory demonstrated. Internal consistency was excellent $(\alpha=0.96)$ and test-retest reliability was stable $(\mathrm{ICC}=0.97)$.

Conclusions: The AR-PAID scale is a valid and reliable instrument for diabetes-distress screening in primary care patients with T2DM that can be used in clinical settings and research. Further research is needed to validate short forms of the AR-PAID scale.

Keywords: diabetes-distress, PAID, primary healthcare, type 2 diabetes, Arabic 


\section{INTRODUCTION}

Diabetes is a global health problem with a prevalence of $9.3 \%$ in 2019. Its prevalence in the Middle East and North Africa region was the highest age-adjusted diabetes prevalence (12.2\%) of all world regions. T2DM accounts for about $90 \%$ of all diabetes cases worldwide (1).

Diabetes-distress is a common comorbid psychological problem with diabetes (36\%) (2), and it reflects the diabetic patient's emotional response to the burden of living with debilitating complications and the greater self-care demands of diabetes (3-6). Diabetes-distress is linked to suboptimal adherence and poor glycemic outcomes (5, 7-10). The American Diabetes Association has recommended screening for diabetesdistress by validated tools at the initial visit of a diabetic patient, at periodic intervals, and when there is a change in disease, treatment, or life circumstances (11).

The PAID scale is an instrument widely used to assess diabetes-distress. It includes 20 items (2). Polonsky et al. had developed this scale for assessing psychosocial adjustment to diabetes in the USA (12-14). Hermanns et al. found that the PAID scale may also be used as a sound-screening instrument for depression in diabetes with a cut-off score of $\geq 33$, which is lower than the normal cut-off score of $\geq 40$ for identifying diabetes-distress (15). The PAID scale is also a useful instrument for assessing several aspects of quality of life in diabetes patients $(16,17)$. The responsiveness of the PAID has also been found; the PAID is able to detect change as demonstrated in seven diabetes intervention studies (18).

Interest in diabetes-distress across countries through the previous two decades means that versions in multiple languages of the PAID instruments are not only desirable, but also necessary in order to compare the findings about diabetes-distress from different languages, cultures, and societies, in addition to the improvement of diabetic care. The original PAID has been translated into several languages successfully, and these versions of it are reliable and valid instruments (19-32).

Previous Arabic studies evaluated the validity and reliability of the 20-item AR-PAID scale in Kuwait and Lebanon. An early study revealed that the AR-PAID was valid and reliable in older adult patients with T2DM, who were recruited from one hospitalbased diabetes unit and one primary diabetic clinic in Kuwait City. Another Arabic study found that the AR-PAID had excellent reliability and an acceptable convergent with HbA1c in Lebanese

\footnotetext{
Abbreviations: AR-PAID, Arabic version of the Problem Areas in Diabetes; BMI, Body Mass Index; CFA, Confirmatory factor analysis; CFI, Comparative fit index; CI, Confidence interval; df, Degrees of freedom; DM, Diabetes mellitus; GAD7, Generalized Anxiety Disorder Scale 7; GFI, Goodness-of-fit index; HbAlc, Glycated hemoglobin; HTN, Hypertension; ICC, Intraclass correlation coefficient; IQR, Interquartile range; PAID, Problem Areas in Diabetes; PAID-1, 1-item Problem Areas in Diabetes; PAID-5, 5-item Problem Areas in Diabetes; PHC, Primary Healthcare; PHQ-9, Patient Health Questionnaire 9; Rho, Spearman's Rank-Order Correlation; RMSEA, Root mean squared error of approximation; SD, Standard deviation; SPSS, Statistical Package for the Social Sciences; SRMR, Standardized root mean square residual; T2DM, Type 2 diabetes mellitus; TLI, Tucker Lewis Index ; WHO, World Health Organization; WHO-5, 5-item World Health Organization Well-Being Index; $\chi^{2}$, Chi-square; CMIN/DF, Ratio of Chisquare value to the degrees of freedom.
}

adults with T2DM, but its construct validity was not assessed $(33,34)$.

Venkataraman et al. displayed the abridged 16-item PAID scale as reliable, valid, and sensitive in Singaporean patients with diabetes (35). Hsu et al. showed that the 8-item short form of the PAID demonstrated sound validity and reliability in Chinese patients with T2DM (36). McGuire et al. developed the 5-item PAID (PAID-5) and 1-item PAID (PAID-1) from the original PAID (37).

In light of the burden of T2DM in Middle East and Arabic countries, increased interest in diabetes-distress, and a lack of studies in validity and reliability of the AR-PAID in primary care, this study was carried out to evaluate the psychometric properties of the AR-PAID in Egyptian patients with T2DM attending PHC settings.

\section{METHODS}

\section{Design, Sampling, and Setting}

A cross-sectional study was conducted on a convenience sample of patients with T2DM from September 2020 to June 2021. The participants were recruited from six rural PHC settings at the Ismailia governorate, affiliated with Egypt's Ministry of Health and Population. Soper's online calculator of a sample size for structural equation models was used to estimate the sample size for a CFA model of a four-factor and the 20 observed variables of the PAID scale (38). The sample size of 173 was enough to detect an expected effect size of 0.3 (a medium effect size) at $5 \%$ alpha error and $90 \%$ power of the study. And in addition, more increase of the sample size to 200 patients to compensate for dropout, guided by the " 10 participants per item" rule of thumb for calculation of the sample size for confirmatory factor analysis (39).

We included participants aged 18 years or older who were diagnosed with T2DM for at least 1 year. An informed consent was obtained from every participant. We excluded patients with a severe mental illness, cognitive impairment, or gestational diabetes. Face-to-face interviews by the second author were used to collect data. A 1-month test-retest on reliability of the ARPAID scale was conducted on 50 participants.

\section{Study Measures and Scales}

Our study's questionnaire consisted of several parts. The initial part included the sociodemographic and clinical characteristics, e.g., age, gender, marital status, family income, occupation, smoking diabetes duration, diabetes treatment, co-morbidities, and diabetes-related complications (e.g., cardiovascular, cerebrovascular, retinopathy, nephropathy, neuropathy, or peripheral vascular complications). The next part of our study's questionnaire included the Arabic versions of the PAID, PHQ-9 $(40,41)$, GAD-7 $(41,42)$, and WHO-5 $(43,44)$ scales.

The original PAID scale was written in English and consisted of 20 items rated on a 5-point Likert scale from 0 to 4 , with 0 indicating no problem and 4 indicating a major problem. The PAID calculates a total score range of 0 to 100 by adding the replies to all 20 items and multiplying the result by 1.25 . The higher the score, the more diabetes-distress, with a score of 40 
or above indicating significant diabetes-distress $(15,45)$. The AR-PAID was obtained from the Joslin Diabetes Center.

The PHQ-9 consists of 9 items, each with a score ranging from 0 (never) to 3 (almost every day), for a total score of 0 to 27. For the detection of major depressive disorder, a cut-off value of $\geq 10$ has an 88 percent sensitivity and an 88 percent specificity (40). The Arabic version of the PHQ-9 has previously shown to be a valid and reliable tool to screen depression in a Saudi sample. Cronbach's alpha was 0.857 . Inter-item correlations range between 0.177 and 0.648 (41).

The GAD-7 is a 7-item anxiety portion that is part of the full GAD. Each GAD item is given a score ranging from 0 (never) to 3 (almost every day), for a total score of 0 to 21 . GAD is indicated by a cut-off value $\geq 10$ ( 89 percent sensitivity and 82 percent specificity) (42). The Arabic version of the GAD-7 demonstrated a sound validity and reliability properties. Cronbach's alpha was 0.763 . Its range of the inter-item correlations was from 0.204 to 0.426. (41).

The WHO-5, which was generated from the WHO-10 (43), is one of the most extensively used questionnaires for assessing subjective psychological wellbeing (46). This scale only has elements that are positively stated. On a 6-point Likert-type scale ranging from 0 (not present) to 5 (constantly present), the degree to which these feelings were present in the previous 2 weeks is scored. The raw score is multiplied by 4 to convert the item scores to a $0-100$ scale (47). The Arabic version of the WHO5 demonstrated validity and reliability in the Lebanese older population (44).

All participants had their anthropometric measurements taken, including their body weight (in $\mathrm{kg}$ ) and height (in $\mathrm{cm}$ ). The BMI was computed by dividing the body weight (in $\mathrm{kg}$ ) by the squared root of the height (in meters). Overweight participants had a BMI of 25-29.9, while obese participants had a BMI of more than $30 \mathrm{~kg} / \mathrm{m}^{2}$. The patient's most recent $\mathrm{HbAlc}$ readings were recorded ( $<8$ weeks prior or 12 weeks after the interview). Adult and older adult patients with controlled glycemic targets were identified by HbA1c levels $<7$ and $7.5 \%$, respectively (11).

\section{Statistical Analysis}

Data management and analyses were performed using the Statistical Package for the Social Sciences (SPSS), version 25.0 (IBM Corporation, NY, USA). Categorical variables were tabulated as frequencies and percentages, while continuous variables were summarized by means and standard deviations (median and interquartile ranges, if not normally distributed). Continuous variables were tested for data normality with the Kolmogorov-Smirnov test.

The AR-PAID was tested for both reliability and validity (construct, convergent, and discriminant). Reliability testing was performed using Cronbach's alpha for internal consistency, as well as ICC for test-retest reliability.

The construct validity of AR-PAID-20 was evaluated in a series of confirmatory factor analyses (CFA), in which we compared the model fit indices of previously proposed models of PAID's factor structure models: the original one-factor of the original PAID, two-factor of the Turkish PAID, three-factor of the Swedish PAID, four-factor of the Dutch PAID, and four-factor of the
Spanish PAID $(13,19,22,24,28)$. The model fit included: (1) the ratio of chi-square statistics $\left[\chi^{2}\right]$ to the degrees of freedom [df] (CMIN/DF) and associated $p$-values; (2) the standardized root mean square residual (SRMR); (3) the root mean squared error of approximation (RMSEA); (4) the comparative fit index (CFI); and (5) the Tucker Lewis Index (TLI). The model fit acceptability was set at CMIN/DF $<3$, SRMR and RMSEA $\leq 0.08$, and CFI and TLI $\geq 0.90$ (27). All CFAs were performed with Mplus software for statistical analysis with Latent variables, version 7.4 (Los Angeles, CA, USA: Muthén and Muthén (48)].

The convergent validity was established by testing for correlations between the AR-PAID and other related scales (e.g., the PHQ-9 for depression and the GAD-7 for anxiety) and the level of glycemic control (HbA1C level). Spearman's correlation was used with the following coefficient values: 00.19 considered very weak, $0.2-0.39$ considered weak, $0.40-0.59$ considered moderate, $0.6-0.79$ considered strong, and $0.8-1.0$ considered very strong correlations (49).

In regards to discriminant validity, the AR-PAID's ability to differentiate between different groups of patients was tested with the Mann-Whitney and Kruskal-Wallis tests, in which the number of groups compared was given. Total AR-PAID scores were compared across patients' groups, such as patients with major depression or generalized anxiety disorder, patients with poor glycemic control, and patients with different demographic and clinical characteristics. All $p<0.05$ were considered statistically significant at a $95 \%$ level of confidence.

\section{RESULTS}

\section{Descriptive Statistics}

Two-hundred patients with T2DM were interviewed in this study. Participants' ages ranged from 20 to 80 years, with a mean of $48.3 \pm 11.4$ years. Twenty-four percentage of them were younger than 40 years. Females made up $60 \%$ of all participants. The majority (76\%) of the participants were married. The mean duration of diabetes was $8.2 \pm 6.2$ years and ranged from 1 to 30 years. Sixty-seven (33.5\%) patients were on insulin-containing regimen. Most (64.5\%) of the participants had one or more diabetes-related complications, which were neuropathy $(50.5 \%)$, retinopathy (36.5\%), foot problems (33.0\%), and nephropathy $(21.5 \%)$. The most common chronic comorbidities were obesity (36.5\%) and hypertension (23.5\%). The mean HbA1c level was $7.9 \pm 0.84 \%$ and ranged from 6 to $14.5 \%$, with only 17 patients (8.5\%) having achieved good glycemic control (Table $\mathbf{1}$ ).

\section{Factor Structure of the AR-PAID}

Five alternative factor structures were compared for the goodness-of-fit indices using confirmatory factor analysis in Table 2. The Belendez's et al. revised four-factor (28) showed the best goodness-of-fit indices $(\mathrm{CMIN} / \mathrm{DF}=2.26, \mathrm{RMSEA}=$ $0.079, \mathrm{SRMR}=0.057, \mathrm{CFI}=0.099$, and TLI 0.988). Accordingly, the factor structure of the Belendez's et al. model (28) is further explained with standardized factor loadings in Table 3. The standardized factor loadings for items in this model were satisfactory and statistically significant and ranged from 0.526 to 1.002 . 
TABLE 1 | Patients' demographic and clinical characteristics $(N=200)$.

\begin{tabular}{|c|c|}
\hline Characteristics & Frequency (\%) \\
\hline Age (years), mean \pm SD (range) & $48.8 \pm 12.1(20-80)$ \\
\hline$<40$ years & $48(24.0 \%)$ \\
\hline $40-59$ & 107 (53.5\%) \\
\hline $60+$ & $45(22.5 \%)$ \\
\hline \multicolumn{2}{|l|}{ Gender } \\
\hline Female & $120(60.0 \%)$ \\
\hline Male & $80(40.0 \%)$ \\
\hline \multicolumn{2}{|l|}{ Marital status } \\
\hline Single & $8(4.0 \%)$ \\
\hline Married & 152 (76.0\%) \\
\hline Divorced/widow & $40(20.0 \%)$ \\
\hline \multicolumn{2}{|l|}{ Education level } \\
\hline Illiterate & $44(22.0 \%)$ \\
\hline Less than secondary & 7 (3.5\%) \\
\hline Secondary & 117 (58.5\%) \\
\hline University and above & $32(16.0 \%)$ \\
\hline \multicolumn{2}{|l|}{ Work status } \\
\hline Not employed/housewives & $92(46.0 \%)$ \\
\hline Employed/business owners/freelancers & $83(41.5 \%)$ \\
\hline Retired & $25(12.5 \%)$ \\
\hline Duration of diabetes, mean \pm SD (range) & $8.2 \pm 6.2(1-30)$ \\
\hline$<5$ years & $67(33.5 \%)$ \\
\hline $5-10$ years & $82(41.0 \%)$ \\
\hline More than 10 years & $51(25.5 \%)$ \\
\hline \multicolumn{2}{|l|}{ Type of antidiabetic medications } \\
\hline Oral hypoglycemics & $133(66.5 \%)$ \\
\hline Insulin-Containing regimens & 67 (33.5\%) \\
\hline \multicolumn{2}{|l|}{ Number of diabetes-related complications } \\
\hline None & $71(35.5 \%)$ \\
\hline Single & $49(24.5 \%)$ \\
\hline Two or more & $80(40.0 \%)$ \\
\hline \multicolumn{2}{|l|}{ Type of diabetes-related complications } \\
\hline Retinopathy & $73(36.5 \%)$ \\
\hline Nephropathy & $43(21.5 \%)$ \\
\hline Neuropathy & $101(50.5 \%)$ \\
\hline Foot problems & $66(33.0 \%)$ \\
\hline Others & $12(6.0 \%)$ \\
\hline \multicolumn{2}{|l|}{ Other chronic comorbidities } \\
\hline Obesity & $73(36.5 \%)$ \\
\hline Hypertension & 47 (23.5\%) \\
\hline Dyslipidemia & $22(11.0 \%)$ \\
\hline $\mathrm{HbA1c} \%$, mean \pm SD (range) & $7.9 \pm 0.84(6.0-14.5)$ \\
\hline \multicolumn{2}{|l|}{ Glycemic control } \\
\hline Controlled & $17(8.5 \%)$ \\
\hline Uncontrolled & $183(91.5 \%)$ \\
\hline
\end{tabular}

\section{Convergent Validity of the AR-PAID}

Table 4 shows the median and interquartile range (IQR) of the AR-PAID and its subscales. Convergent validity was confirmed by significant moderate-to-strong correlations between the total scores of the AR-PAID with: the PHQ-9 scale for depression (rho $=0.71, p<0.001$ ), the GAD-7 scale for anxiety ( $r h o=0.50, p$
$<0.001$ ), and the WHO-5 wellbeing index (rho $=-0.69, p<$ 0.001). The total score of the AR-PAID also showed a significant but weak correlation with $\mathrm{HbA1}$ c levels (rho $=0.38, p<0.001$ ). The AR-PAID subscales showed significant correlations with all measures, with correlation coefficients ranging from 0.15 to 0.68 . All AR-PAID subscales had their highest correlations with the PHQ-9 scale and WHO-5 wellbeing index, while their weakest correlations existed with HbA1C levels (Table 4).

\section{Discriminant Validity of the AR-PAID}

The AR-PAID scale showed the ability to discriminate between diabetes-distress among patients with different demographic and clinical characteristics (Figure 1). Female patients and patients with older age or longer disease duration showed significantly higher total scores of the AR-PAID. Patients on insulin-containing regimen also had significantly higher total AR-PAID scores compared to patients on oral hypoglycemic agents. Increasing the number of diabetes-related complications was significantly associated with higher total AR-PAID scores in contrast to those with no complications. Patients with obesity, hypertension, or dyslipidemia also showed significantly higher total AR-PAID scores compared to patients without comorbidities. In contrast, there was no significant difference in AR-PAID total scores between patients with controlled diabetes and those with uncontrolled $(p=0.145)$. Known-group validity was confirmed by the statistically significant differences in ARPAID total scores between patients with and without depression (PHQ-9 $\geq 10$ ), patients with and without generalized anxiety disorder (GAD-7 $\geq 10$ ), and patients with and without poor wellbeing (WHO-5 index $\leq 50$ ).

\section{Reliability of the AR-PAID}

Cronbach's alpha for the total AR-PAID scale was 0.96, while it ranged from 0.65 to 0.96 for the AR-PAID subscales. The subscales of emotional and treatment problems showed the highest Cronbach's alpha. Test-retest reliability was investigated in 50 patients and revealed satisfactory significant ICCs. The total AR-PAID scale had an ICC of 0.97 (95\% CI: 0.61-0.83, $p<0.001$ ), while the ICC of the AR-PAID subscales ranged from 0.92 to 0.97 (Table 5).

\section{DISCUSSION}

We evaluated the psychometric properties of the AR-PAID in Egyptian primary care patients with T2DM. We found that the AR-PAID had a four-factor structure, had a satisfied convergent and discrimination validity, and was internally consistent with stable test-retest reliability.

A CFA supported a four-factor structure of the AR-PAID similar to those found in the Spanish and Kinyarwanda versions of the PAID $(28,31)$. A CFA of the AR-PAID also demonstrated marginal fit to the four-factor model of the Duch PAID, as well as the three-factor model of the Swedish PAID. The four-model of the Spanish PAID included item 15 ("feeling unsatisfied with your diabetes physician") in a social support problems subscale, while the four-factor model of the Duch PAID included this item in a treatment problems subscale. The Greek and Malaysian versions 
TABLE 2 | Comparisons of fit indices of different AR-PAID factor solutions ( $N=200)$.

\begin{tabular}{|c|c|c|c|c|c|c|c|c|}
\hline \multirow{2}{*}{$\begin{array}{l}\text { Factor solution } \\
\text { models }\end{array}$} & \multirow[t]{2}{*}{ Factors (items included) } & \multicolumn{7}{|c|}{ Goodness-of-Fit indices } \\
\hline & & DF & $\mathrm{x}^{2}$ & CMIN/DF & RMSEA & CFI & TLI & SRMR \\
\hline One-Factor (13) & Problem areas in diabetes (items 1-20) & 164 & $714.9^{\star \star}$ & 4.36 & 0.127 & 0.972 & 0.969 & 0.078 \\
\hline Two-Factor (24) & $\begin{array}{l}\text { - Diabetes distress (15 items): 1-14, } 19 \\
\text { - Lack of support (5 items): 15-18, } 20\end{array}$ & 164 & $678.4^{\star \star}$ & 4.14 & 0.123 & 0.974 & 0.971 & 0.077 \\
\hline Three-Factor (22) & $\begin{array}{l}\text { - Emotional problems (15 items): 3, 6-10, } \\
\text { 12-14, 16, 19, } 20 \\
\text { - Treatment problems (2 items): 1, } 2 \\
\text { - Lack of support (3 items): 15, 17, } 18\end{array}$ & 164 & $481.0^{\star \star}$ & 2.93 & 0.097 & 0.984 & 0.982 & 0.059 \\
\hline Four-Factor (19) & $\begin{array}{l}\text { - Emotional problems (12 items): 3, 6-10, } \\
\text { 12-14, 16, 19-20 } \\
\text { - Treatment problems (3 items): 1, 2, } 15 \\
\text { - Food-related problems (3 items): 4, 5, } 11 \\
\text { - Lack of support (2 items): } 17,18\end{array}$ & 164 & $391.1^{\star \star}$ & 2.38 & 0.083 & 0.988 & 0.987 & 0.059 \\
\hline $\begin{array}{l}\text { Revised four-factor } \\
\text { (28) }\end{array}$ & $\begin{array}{l}\text { - Emotional problems (12 items): 3, 6-10, } \\
\text { 12-14, 16, 19-20 } \\
\text { - Treatment problems (2 items): 1, } 2 \\
\text { - Food problems ( } 3 \text { items): } 4,5,11 \\
\text { - Lack of support (3 items): } 15,17,18\end{array}$ & 164 & $371.2^{\star \star}$ & 2.26 & 0.079 & 0.990 & 0.988 & 0.057 \\
\hline
\end{tabular}

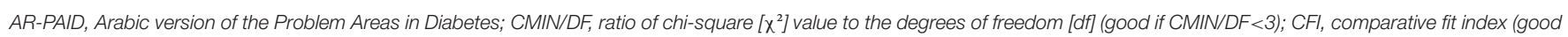

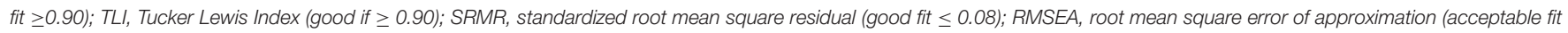
$\leq 0.08)$.

${ }^{* \star}$ Statistically significant $p(<0.001)$.

of the PAID had a three-factor structure, both of which had different components of subscales compared to each other and to the Swedish version of the PAID. The Brazilian and German versions of the PAID fitted the four-factor structure, while the Kuwaiti AR-PAID fitted the five-factor structure (19, 20, 22, 26, 28-30, 33).

Our CFA was inadequately fit with one-factor and two-factor models. A one-factor structure had been found in the original version of the PAID, which was demonstrated by Welch et al. in addition to the Brazilian, Taiwanese, and Polish versions of the PAID $(13,20,23,32)$. A two-factor structure was reported in the Icelandic and Turkish versions of the PAID $(21,24)$. Miller and Elasy also identified a two-factor structure of the PAID in southern rural African-American women with T2DM (50). The Korean PAID was marginally fit with one-factor to four-factor models (27).

These discrepancies of the structures of these versions of the PAID could be related to differences in the cultural and clinical characteristics of the participants, the existing health care system and diabetic care services that might affect patients' perceptions about diabetes-distress, the use of exploratory factor analysis in some previous studies, and the use of different methods.

Convergent validity of the total AR-PAID scale was supported by the moderate-to-strong positive correlations with the PHQ-9 and GAD-7 scores, by the strong negative correlation with the WHO- 5 score, and by the weak positive correlation with HbAlc levels. The AR-PAID displayed strong correlation with the PHQ9, whereas both previous studies found moderate correlation with the PHQ-9 $(32,51,52)$. The AR-PAID displayed moderate correlation with the GAD-7, which was similar to what was found in an Australian study (52). The AR-PAID correlated moderately and negatively with the WHO-5, and those results were consistent with those found in validation studies of the Turkish and Polish versions of the PAID $(24,32)$.

The total score of the AR-PAID has a weak positive correlation with HbA1c levels, similarly observed in previous studies $(13,19$, $22,23,25-28,30,32)$. However, other studies found insignificant associations between versions of the PAID and HbA1c levels $(20,24,29)$. This association was not assessed in the Kinyarwanda PAID, as the HbAlc test was not assessed systematically in the Rwandan patients with diabetes (31).

In our study, the emotional problem subscale had the highest correlation with depression, anxiety, wellbeing, and glycemic control (HbA1c levels) compared with other subscales. These findings might be related to the importance and relevance of the emotional problems subscale for evaluating the pivotal content of diabetes-stress $(28,37)$. Therefore, further study is needed to assess the psychometric properties of the short version of the AR-PAID.

The total AR-PAID scale had an excellent internal consistency ( $\alpha=0.96$ ), which was in line with the reported findings from the original, Duch, Brazilian, Icelandic, Swedish, Turkish, Norwegian, Greek, Korean, Spanish, German, Malaysian, and Polish versions of the PAID ( $\alpha$ ranged 0.90-0.96) (12, 13, 19-22, $24-30,32)$. The Kinyarwanda version of the PAID demonstrated a good internal consistency $(\alpha=0.88)$ (31), while the internal consistency analysis was not reported in the Taiwanese version of the PAID (23).

We found that the emotional problems subscale had demonstrated the highest Cronbach's alpha. This unsurprising finding might be the result of its homogenous construct, as well as inclusion of more items in this subscale: twelve items, compared 
TABLE 3 | Factor loadings of the revised 4-factor model of the AR-PAID $(N=200)$.

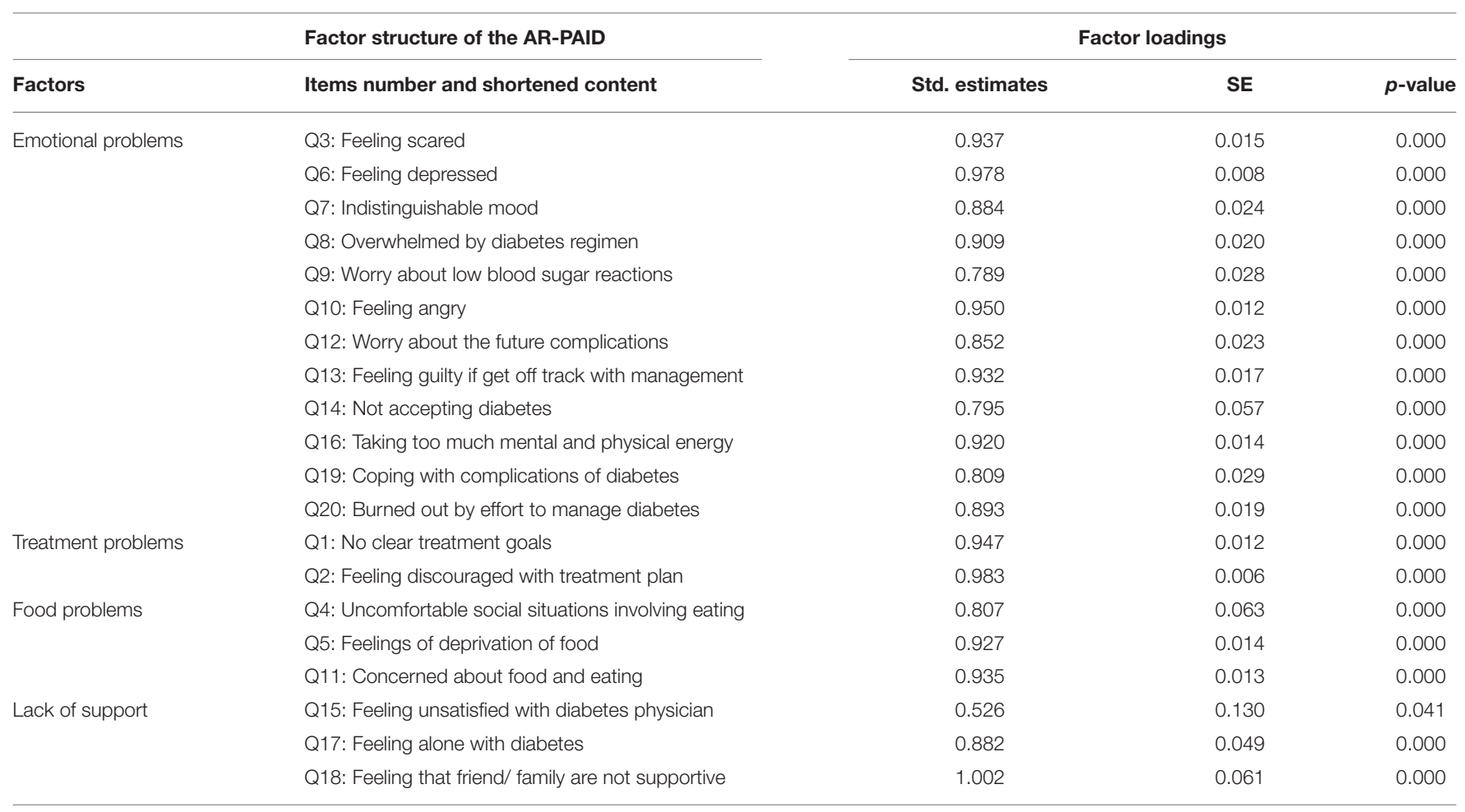

\section{Goodness-of-Fit indices}

$\begin{array}{lc}\text { Model fit, } \chi^{2}(\mathrm{df}), p \text {-value } & 371.2(164),<0.001^{*} \\ \text { CMIN/DF }\left(\chi^{2} / \mathrm{df}\right) & 2.26 \\ \text { CFI } & 0.990 \\ \text { TLI } & 0.988 \\ \text { SRMR } & 0.057 \\ \text { RMSEA } & 0.079\end{array}$

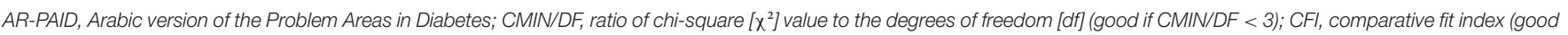

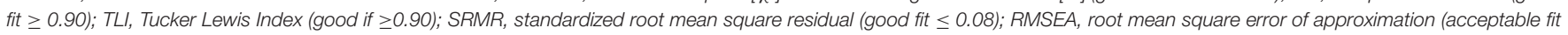
$\leq 0.08)$; SE, standard error.

${ }^{\star}$ Statistically significant at $p<0.001$.

TABLE 4 | Correlations of the AR-PAID and its subscales with other measures of effect and HbA1C $(N=200)$.

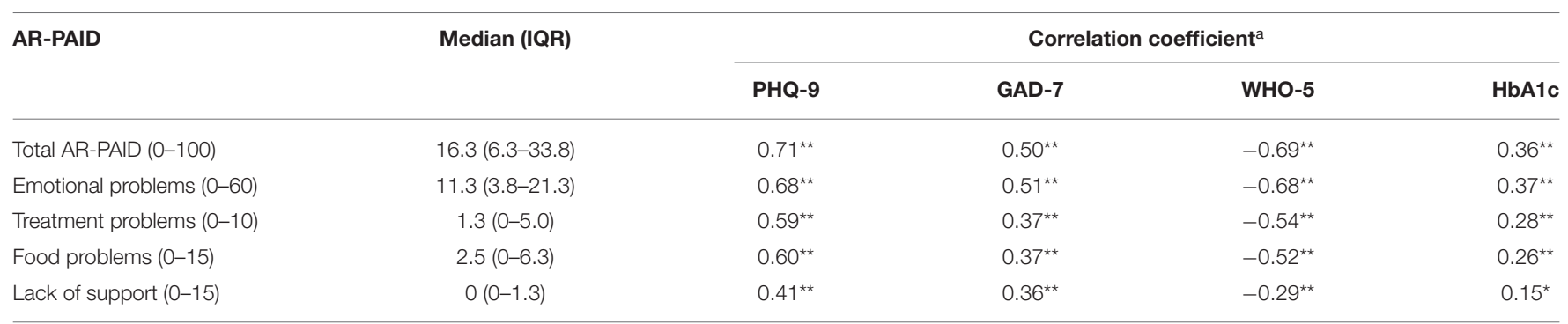

AR-PAID, Arabic version of the Problem Areas in Diabetes; GAD-7, Generalized Anxiety Disorder Scale 7; HbA1c, Glycated hemoglobin; PHQ-9, Patient Health Questionnaire 9; WHO-5, 5-item World Health Organization Well-Being Index.

a Spearman's Correlation. IQR: Interquartile range.

${ }^{* *} p<0.001,{ }^{*} p<0.05$. 

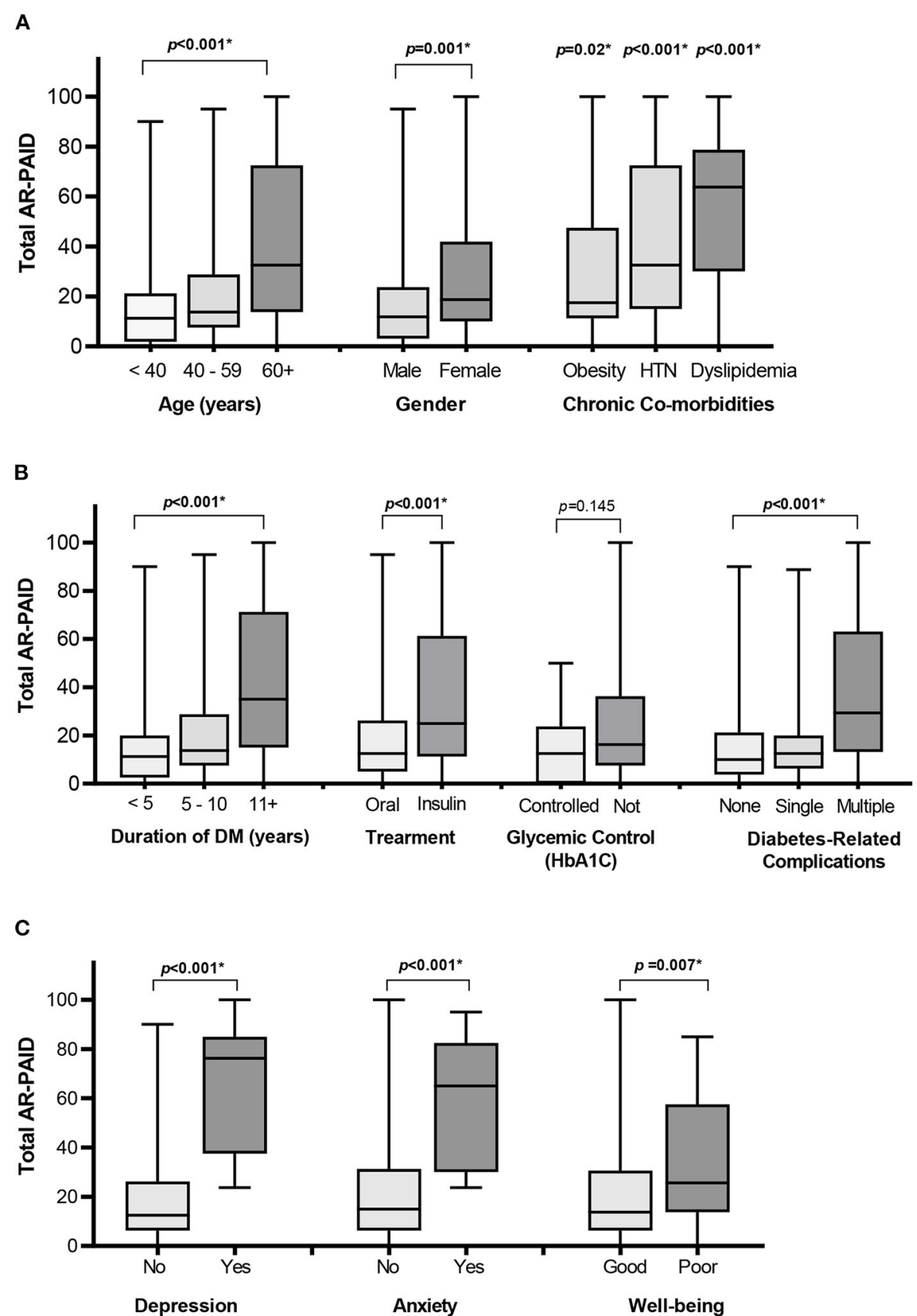

FIGURE 1 | Distribution of total AR-PAID score by patients' demographic and clinical characteristics: age, gender, and co-morbidities (A), diabetes-related characteristics (B), and depression, anxiety, and WHO-wellbeing scales (C). *Indicates a statistically significant $p<0.05$. DM, diabetes mellitus; HTN, hypertension; WHO-5, 5-item World Health Organization Well-Being Index.

to other subscales. The treatment problems subscale also had an excellent internal consistency ( $\alpha=0.94)$, which is inconsistent with the findings of the Korean PAID $(\alpha=0.54)$. Lee et al. had explained their finding as a result of the relative heterogeneity of its three items, which may be related to the presence of item 15 (27).
The total AR-PAID scale was found to have good 1-month test-retest reliability (ICC $=0.97$ ), indicating the stability of the scale. Previous studies reported a satisfactory stability of the total PAID scale $(19,23,25,27)$, whereas the test-retest of it was not assessed in other studies $(12,13,20-22,24,28-34,50)$. We found that the AR-PAID subscales demonstrated satisfactory stability 
TABLE 5 | Internal consistency and test-retest reliabilities of the AR-PAID ( $N=200)$.

\begin{tabular}{|c|c|c|c|c|}
\hline \multirow[t]{2}{*}{ Items of the AR-PAID (score range) } & \multirow[t]{2}{*}{ Number of items } & \multirow[t]{2}{*}{ Cronbach $\alpha$} & \multicolumn{2}{|c|}{ Test-Retest reliability $(n=50)$} \\
\hline & & & ICC & $95 \% \mathrm{Cl}$ \\
\hline Emotional problems (0-60) & 12 & 0.96 & 0.93 & $0.86-0.96$ \\
\hline Treatment problems (0-10) & 2 & 0.94 & 0.97 & $0.88-0.99$ \\
\hline Food problems (0-15) & 3 & 0.79 & 0.92 & $0.52-0.97$ \\
\hline Lack of support (0-15) & 3 & 0.65 & 0.96 & $0.89-0.98$ \\
\hline Total AR-PAID (0-100) & 20 & 0.96 & 0.97 & $0.94-0.98$ \\
\hline
\end{tabular}

AR-PAID, Arabic version of the problem areas in diabetes; SD, Standard deviation; ICC, intra-class correlation; Cl, confidence interval.

(ICC ranged 0.92-0.97). Snoek et al. (19) and Lee et al. (27) had both reported instability of their treatment problems subscale.

Our results provided sound support for the discriminant validity of the AR-PAID in terms of determining differences in diabetes-distress with patients' sociodemographic and clinical characteristics. We found older people with T2DM showed significantly higher diabetes-distress scores than other age groups, which might have been explained by how older people reported being usually concerned about the presence and seriousness of diabetes-related complications, dealing with complications, and controlling diabetes, as well as being anxious and having guilty sensations if they are not able to achieve good glycemic targets. However, other studies found that younger patients had higher scores on the PAID $(19,20,24,32)$. Female patients had significantly higher AR-PAID total scores than male patients. Similar findings have been found in previous studies $(19,20,24-26,32,33)$.

In the current study, patients with longer disease duration and those who are on insulin-containing regimen reported significantly higher AR-PAID scores, which was similar to the findings of a Greek study (26). Patients with an increasing number of the diabetes-related complications showed significantly higher AR-PAID total scores, and this finding was in line with the findings from a validation study of the Turkish PAID (24). We did not find an association between the AR-PAID total scores and achieving good glycemic targets, though this might be due to the relatively few participants who were able to achieve good glycemic targets. Knowngroup validity was supported by the significant associations of the AR-PAID total scores with depression, anxiety, and general wellbeing.

To the best of our knowledge, this is the first study that assessed the validity and reliability of the AR-PAID among PHC patients in the Middle East and North Africa region. Our study included only PHC patients with T2DM, which limited our ability to generalize the results to patients with type 1 diabetes. Our study included a relatively small number of patients who were able to achieve good glycemic control, which restricted the group validity on this clinical variable. Lastly, the crosssectional design of our study could not assess the responsiveness of this instrument.

\section{CONCLUSIONS}

The AR-PAID scale is a valid and reliable instrument for assessing diabetes-distress among Egyptian PHC patients with T2DM. Further studies are needed to assess the responsiveness of the AR-PAID, to assess cultural adaptations of the AR-PAID in other Arabic-speaking patients with diabetes, and to assess the validity and reliability of short forms of the AR-PAID.

\section{DATA AVAILABILITY STATEMENT}

The raw data supporting the conclusions of this article will be made available by the authors, upon reasonable request.

\section{ETHICS STATEMENT}

The studies involving human participants were reviewed and approved by Ethics Committee of Faculty of Medicine, Suez Canal University, Ismailia, Egypt (Ref No. 4277/2020). The patients/participants provided their written informed consent to participate in this study.

\section{AUTHOR CONTRIBUTIONS}

HS commenced the idea of this study, participated in the designing the study, wrote the manuscript draft, and approved the final version of the manuscript. SM participated in the designing of this study, collected the data, revised the manuscript, and approved the final version of the manuscript. SE participated in the designing of this study, analyzed the data, revised the manuscript, and approved the final version of the manuscript. MM and JS participated in the designing of this study, revised the manuscript, and approved the final version of the manuscript. AF designed this study, analyzed the data, revised the manuscript, and approved the final version of the manuscript. All authors contributed to the article and approved the submitted version.

\section{ACKNOWLEDGMENTS}

Deepest appreciation for the primary care patients with T2DM who participated in our study. 


\section{REFERENCES}

1. International Diabetes Federation. IDF Diabetes Atlas. $9^{\text {th }}$ ed. Brussels: International Diabetes Federation (2019). Available online at: https:// www.diabetesatlas.org/upload/resources/material/20200302_133351_ IDFATLAS9e-final-web.pdf

2. Perrin NE, Davies MJ, Robertson N, Snoek FJ, Khunti K. The prevalence of diabetes-specific emotional distress in people with type 2 diabetes: a systematic review and meta-analysis. Diabet Med. (2017) 34:150820. doi: $10.1111 /$ dme. 13448

3. Fisher L, Glasgow RE, Strycker LA. The relationship between diabetes distress and clinical depression with glycemic control among patients with type 2 diabetes. Diabetes Care. (2010) 33:1034-6. doi: 10.2337/dc09-2175

4. Fisher L, Hessler DM, Polonsky WH, Mullan J. When is diabetes distress clinically meaningful?: establishing cut points for the diabetes distress scale. Diabetes Care. (2012) 35:259-64. doi: 10.2337/dc11-1572

5. Snoek FJ, Bremmer MA, Hermanns N. Constructs of depression and distress in diabetes: time for an appraisal. Lancet Diabetes Endocrinol. (2015) 3:45060. doi: 10.1016/S2213-8587(15)00135-7

6. Fisher L, Polonsky WH, Hessler D. Addressing diabetes distress in clinical care: a practical guide. Diabet Med. (2019) 36:803-12. doi: 10.1111/dme. 13967

7. Fisher L, Skaff MM, Mullan JT, Arean P, Mohr D, Masharani U, et al. Clinical depression versus distress among patients with type 2 diabetes: not just a question of semantics. Diabetes Care. (2007) 30:542-8. doi: 10.2337/dc06-1614

8. Delahanty LM, Grant RW, Wittenberg E, Bosch JL, Wexler DJ, Cagliero E, et al. Association of diabetes-related emotional distress with diabetes treatment in primary care with type 2 diabetes. Diabet Med. (2007) 24:4854. doi: 10.1111/j.1464-5491.2007.02028.x

9. Fisher L, Mullan JT, Arean P, Glasgow RE, Hessler D, Masharani U. Diabetes distress but not clinical depression or depressive symptoms is associated with glycemic control in both cross-sectional and longitudinal analyses. Diabetes Care. (2010) 33:23-8. doi: 10.2337/dc09-1238

10. Aikens JE. Prospective associations between emotional distress and poor outcomes in type 2 diabetes. Diabetes Care. (2012) 35:24728. doi: $10.2337 / \mathrm{dc} 12-0181$

11. American Diabetes Association. Standards of medical care in diabetes-2020 abridged for primary care providers. Clin Diabetes. (2020) 38:1038. doi: $10.2337 / \mathrm{cd} 20$-as01

12. Polonsky WH, Anderson BJ, Lohrer PA, Welch G, Jacobson AM, Aponte JE, et al. Assessment of diabetes-related distress. Diabetes Care. (1995) 18:75460. doi: 10.2337/diacare.18.6.754

13. Welch GW, Jacobson AM, Polonsky WH. The problem areas in diabetes scale. An evaluation of its clinical utility. Diabetes Care. (1997) 20:7606. doi: $10.2337 /$ diacare. 20.5 .760

14. Polonsky WH, Fisher L, Earles J, Dudl RJ, Lees J, Mullan J, et al. Assessing psychosocial distress in diabetes: development of the diabetes distress scale. Diabetes Care. (2005) 28:626-31. doi: 10.2337/diacare.28.3.626

15. Hermanns N, Kulzer B, Krichbaum M, Kubiak T, Haak T. How to screen for depression and emotional problems in patients with diabetes: comparison of screening characteristics of depression questionnaires, measurement of diabetes-specific emotional problems and standard clinical assessment. Diabetologia. (2006) 49:469-77. doi: 10.1007/s00125-005-0094-2

16. Polonsky WH. Understanding and assessing diabetes-specific quality of life. Diabetes Spectr. (2000) 13:36-41.

17. Polonsky WH. Emotional and quality-of-life aspects of diabetes management. Curr Diab Rep. (2002) 2:153-9. doi: 10.1007/s11892-002-0075-5

18. Welch G, Weinger K, Anderson B, Polonsky WH. Responsiveness of the problem areas in diabetes (PAID) questionnaire. Diabet Med. (2003) 20:6972. doi: 10.1046/j.1464-5491.2003.00832.x

19. Snoek FJ, Pouwer F, Welch GW, Polonsky WH. Diabetes-related emotional distress in Dutch and U.S. diabetic patients: cross-cultural validity of the problem areas in diabetes scale. Diabetes care. (2000) 23:13059. doi: $10.2337 /$ diacare.23.9.1305

20. Gross CC, Scain SF, Scheffel R, Gross JL, Hutz CS. Brazilian version of the problem areas in diabetes scale (B-PAID): validation and identification of individuals at high risk for emotional distress. Diabetes Res Clin Pract. (2007) 76:455-9. doi: 10.1016/j.diabres.2006.09.022
21. Sigurdardottir AK, Benediktsson R. Reliability and validity of the icelandic version of the problem area in diabetes (PAID) scale. Int J Nurs Stud. (2008) 45:526-33. doi: 10.1016/j.ijnurstu.2006.10.008

22. Amsberg S, Wredling R, Lins PE, Adamson U, Johansson UB. The psychometric properties of the Swedish version of the problem areas in diabetes scale (Swe-PAID-20): scale development. Int J Nurs Stud. (2008) 45:1319-28. doi: 10.1016/j.ijnurstu.2007.09.010

23. Huang MF, Courtney M, Edwards H, McDowell J. Validation of the Chinese version of the problem areas in diabetes (PAID-C) scale. Diabetes Care. (2010) 33:38-40. doi: 10.2337/dc09-0768

24. Huis In 't Veld EM, Makine C, Nouwen A, Karşidag C, Kadioglu P, Karşidag K, et al. Validation of the Turkish version of the problem areas in diabetes scale. Cardiovasc Psychiatry Neurol. (2011) 2011:315068. doi: 10.1155/2011/315068

25. Graue M, Haugstvedt A, Wentzel-Larsen T, Iversen MM, Karlsen B, Rokne B. Diabetes-related emotional distress in adults: reliability and validity of the Norwegian versions of the problem areas in diabetes scale (PAID) and the diabetes distress scale (DDS). Int J Nurs Stud. (2012) 49:17482. doi: 10.1016/j.ijnurstu.2011.08.007

26. Papathanasiou A, Koutsovasilis A, Shea S, Philalithis A, Papavasiliou S, Melidonis A, et al. The problem areas in diabetes (PAID) scale: psychometric evaluation survey in a Greek sample with type 2 diabetes. J Psychiatr Ment Health Nurs. (2014) 21:345-53. doi: 10.1111/j.1365-2850.2012.01875.x

27. Lee EH, Lee YW, Lee KW, Kim YS, Nam MS. Measurement of diabetes-related emotional distress using the problem areas in diabetes scale: psychometric evaluations show that the short form is better than the full form. Health Qual Life Outcomes. (2014) 12:142. doi: 10.1186/s12955-014-0142-Z

28. Beléndez M, Hernández-Mijares A, Marco J, Domínguez JR, Pomares FJ. Validation of the Spanish version of the problem areas in diabetes (PAID-SP) scale. Diabetes Res Clin Pract. (2014) 106:e93-5. doi: 10.1016/j.diabres.2014.09.012

29. Schmitt A, Reimer A, Kulzer B, Haak T, Ehrmann D, Hermanns N. How to assess diabetes distress: comparison of the problem areas in diabetes scale (PAID) and the diabetes distress scale (DDS). Diabet Med. (2016) 33:83543. doi: $10.1111 / \mathrm{dme} .12887$

30. Jannoo Z, Yap BW, Khan NM, Farcomeni A. Assessing diabetes distress among type 2 diabetes mellitus in malaysia using the problem areas in diabetes scale. Value Health Reg Issues. (2019) 18:159-64. doi: 10.1016/j.vhri.2019.03.004

31. Lygidakis C, Uwizihiwe JP, Bia M, Kallestrup P, Dukundane D, AsiimweKateera B, et al. Cultural adaptation and psychometric evaluation of the Kinyarwanda version of the problem areas in diabetes (PAID) questionnaire. Health Qual Life Outcomes. (2021) 19:183. doi: 10.1186/s12955-021-01821-w

32. Cichoń E, Kiejna A, Gondek TM, Obrebski M, Sutkowska E, Lloyd CE, et al. PAID-PL-The polish version of the problem areas in diabetes scale: perfect reliability and a one-factor structure. Diabetes Metab Syndr Obes. (2021) 14:4433-41. doi: 10.2147/DMSO.S322850

33. Alragum, SN, Hack LM. Health-Related Quality of Life in Kuwait: Validation of Generic and Disease-Specific Measures. (Doctor of Philosophy thesis), Philadelphia: Temple University (2008).

34. Sukkarieh OA, Howard E, Babington L, Badr L. The Relationship Among Diabetes Self-Care, Psychological Adjustment, Social Support and Glycemic Control in the Lebanese Population With Type 2 Diabetes Mellitus?. (Doctor of Philosophy thesis), Boston: Northeastern University-Bouvé College of Health Sciences, School of Nursing (2011).

35. Venkataraman K, Tan LS, Bautista DC, Griva K, Zuniga YL, Amir $M$, et al. Psychometric Properties of the problem areas in diabetes (PAID) instrument in Singapore. PLoS ONE. (2015) 10:e0136759. doi: 10.1371/journal.pone.0136759

36. Hsu HC, Chang YH, Lee PJ, Chen SY, Hsieh CH, Lee YJ, et al. Developing and psychometric testing of a short-form problem areas in diabetes scale in chinese patients. J Nurs Res. (2013) 21:212-8. doi: 10.1097/01.jnr.0000432048.31921.e2

37. McGuire BE, Morrison TG, Hermanns N, Skovlund S, Eldrup E, Gagliardino J, et al. Short-form measures of diabetes-related emotional distress: the problem areas in diabetes scale (PAID)-5 and PAID-1. Diabetologia. (2010) 53:669. doi: 10.1007/s00125-009-1559-5

38. Soper DS. A-priori Sample Size Calculator for Structural Equation Models [Software]. (2021). Available online at: https://www.danielsoper.com/statcalc 
39. Boateng GO, Neilands TB, Frongillo EA, Melgar-Quiñonez HR, Young SL. Best practices for developing and validating scales for health, social, and behavioral research: a primer. Front Public Health. (2018) 6:149. doi: 10.3389/fpubh.2018.00149

40. Kroenke K, Spitzer RL, Williams JB. Validity of a brief depression severity measure. J Gen Intern Med. (2001) 16:60613. doi: 10.1046/j.1525-1497.2001.016009606.x

41. AlHadi AN, AlAteeq DA, Al-Sharif E, Bawazeer HM, Alanazi H, AlShomrani AT, et al. An Arabic translation, reliability, and validation of patient health questionnaire in a Saudi sample. Ann Gen Psychiatry. (2017) 16:32. doi: 10.1186/s12991-017-0155-1

42. Spitzer RL, Kroenke K, Williams JB, Löwe B. A brief measure for assessing generalized anxiety disorder: the GAD-7. Arch Intern Med. (2006) 166:10927. doi: 10.1001/archinte.166.10.1092

43. Bech P, Gudex C, Staehr Johansen K. The WHO (Ten) well-being index: validation in diabetes. Psychother Psychosom. (1996) 65:18390. doi: 10.1159/000289073

44. Sibai AM, Chaaya M, Tohme RA, Mahfoud Z, Al-Amin H. Validation of the Arabic version of the 5-item WHO well being index in elderly population. Int J Geriatr Psychiatry. (2009) 24:106-7. doi: 10.1002/gps.2079

45. Snoek FJ, Kersch NY, Eldrup E, Harman-Boehm I, Hermanns N, Kokoszka A, et al. Monitoring of individual needs in diabetes (MIND): baseline data from the cross-national diabetes attitudes, wishes, and needs (DAWN) MIND study. Diabetes Care. (2011) 34:601-3. doi: 10.2337/dc10-1552

46. Topp CW, Østergaard SD, Søndergaard S, Bech P. The WHO-5 well-being index: a systematic review of the literature. Psychother Psychosom. (2015) 84:167-76. doi: 10.1159/000376585

47. Hajos TR, Pouwer F, Skovlund SE, Den Oudsten BL, Geelhoed-Duijvestijn $\mathrm{PH}$, Tack CJ, et al. Psychometric and screening properties of the WHO-5 wellbeing index in adult outpatients with type 1 or type 2 diabetes mellitus. Diabet Med. (2013) 30:e63-9. doi: 10.1111/dme.12040

48. Muthén, LK, Muthén BO. Mplus User's Guide: Statistical Analysis With Latent Variables, User's Guide. Muthén \& Muthén (2017). Available online at: https://www.statmodel.com/download/usersguide/MplusUserGuideVer_ 8.pdf (accessed December 16, 2021).
49. BMJ Publishing Group. Correlation and Regression. Available online at: http:// www.bmj.com/about-bmj/resources-readers/publications/statistics-squareone/11-correlation-and-regression (accessed December 16, 2021).

50. Miller ST, Elasy TA. Psychometric evaluation of the problem areas in diabetes (PAID) survey in Southern, rural African American women with type 2 diabetes. BMC Public Health. (2008) 8:70. doi: 10.1186/14712458-8-70

51. Reddy J, Wilhelm K, Campbell L. Putting PAID to diabetes-related distress: the potential utility of the problem areas in diabetes (PAID) scale in patients with diabetes. Psychosomatics. (2013) 54:44-51. doi: 10.1016/j.psym.2012. 08.004

52. Fenwick EK, Rees G, Holmes-Truscott E, Browne JL, Pouwer F, Speight J. What is the best measure for assessing diabetes distress? A comparison of the problem areas in diabetes and diabetes distress scale: results from diabetes MILES-Australia. J Health Psychol. (2018) 23:667-80. doi: 10.1177/1359105316642006

Conflict of Interest: The authors declare that the research was conducted in the absence of any commercial or financial relationships that could be construed as a potential conflict of interest.

Publisher's Note: All claims expressed in this article are solely those of the authors and do not necessarily represent those of their affiliated organizations, or those of the publisher, the editors and the reviewers. Any product that may be evaluated in this article, or claim that may be made by its manufacturer, is not guaranteed or endorsed by the publisher.

Copyright (c) 2022 Sayed Ahmed, Mohamed, Elotla, Mostafa, Shah and Fouad. This is an open-access article distributed under the terms of the Creative Commons Attribution License (CC BY). The use, distribution or reproduction in other forums is permitted, provided the original author(s) and the copyright owner(s) are credited and that the original publication in this journal is cited, in accordance with accepted academic practice. No use, distribution or reproduction is permitted which does not comply with these terms. 